\title{
Palpitations following regular ibuprofen dosing in a 13-year-old girl: a case report
}

\author{
Robert J Douglas
}

\begin{abstract}
Introduction: The sensation of palpitations may either be the initial or the only symptom of cardiac arrhythmia. We describe a case of an apparent clear temporal relationship between standard ibuprofen dosing and palpitations. A review of the medical literature revealed this to be, to the best of our knowledge, the first reported case of this type.

Case presentation: A 13-year-old Caucasian girl initially presented to our clinic with hamstring tendinitis. She was commenced on a medication regimen of paracetamol and ibuprofen. After the third ibuprofen dose, she experienced palpitations. These were associated with lower chest and/or upper abdominal discomfort, and a feeling of being hot and sweaty. Her symptoms ceased upon the cessation of ibuprofen therapy.

Conclusion: Cardiac arrhythmia is a potentially fatal disorder that may exhibit heart palpitations as its initial (or only) symptom. The prompt recognition of the cause of the symptom can reduce mortality and morbidity associated with any underlying pathological processes. There is a need to investigate cases of recurrent palpitations so as to exclude underlying structural cardiac pathology and/or abnormal cardiac rhythm.
\end{abstract}

\section{Introduction}

Tendinitis, or the inflammation of a tendon, is a musculoskeletal disorder that is commonly seen in patients in general practice clinics. It is usually treated with a combination of rest, simple analgesics, a non-steroidal antiinflammatory drug (NSAID) and physical rehabilitation. In this case report, we describe a case of cardiac palpitations that temporally appears to be caused by standard ibuprofen dosing, an event that has not been previously described in the medical literature.

\section{Case presentation}

A 13-year-old Caucasian girl weighing $45 \mathrm{~kg}$ was examined in a general practice clinic with a short history of left posterior thigh pain. There was no obvious precipitant condition for her complaint. She reported no significant medical history, took no medications and had no allergies. There was also no significant family medical history. A clinical diagnosis of left hamstring tendinitis was made. She was commenced on a regimen of

Correspondence: rabs01@hotmail.com

Seaton Medical and Specialist Centre, 175 Trimmer Parade, Seaton, South Australia, 5023, Australia paracetamol $1 \mathrm{~g}$ qid prn, ibuprofen $400 \mathrm{mg}$ tds, and a gentle exercise and stretching program.

Shortly after taking the third $400 \mathrm{mg}$ dose of ibuprofen, she felt hot and sweaty, and reported a sensation of palpitations. There was associated lower chest and upper abdominal discomfort, but she felt no frank chest or abdominal pain. No other symptoms, in particularly those suggestive of either an allergic reaction or an acute coronary syndrome, were noted.

Our patient continued with ibuprofen treatment for another day, with exacerbation of her symptoms after each ibuprofen dose. After informing her mother of her symptoms, her ibuprofen medication was discontinued. Her symptoms ceased thereafter.

Upon clinical review a few days later, she was found well with a pulse of 70. An electrocardiogram (ECG) test demonstrated sinus arrhythmia with no abnormality (Figure 1). Her echocardiography result was normal. Subsequent review by a pediatric cardiologist elicited no abnormality. Further review in the clinic determined that her symptoms had not recurred. After discussion with our patient and her mother, a decision was made to not rechallenge her with ibuprofen. 


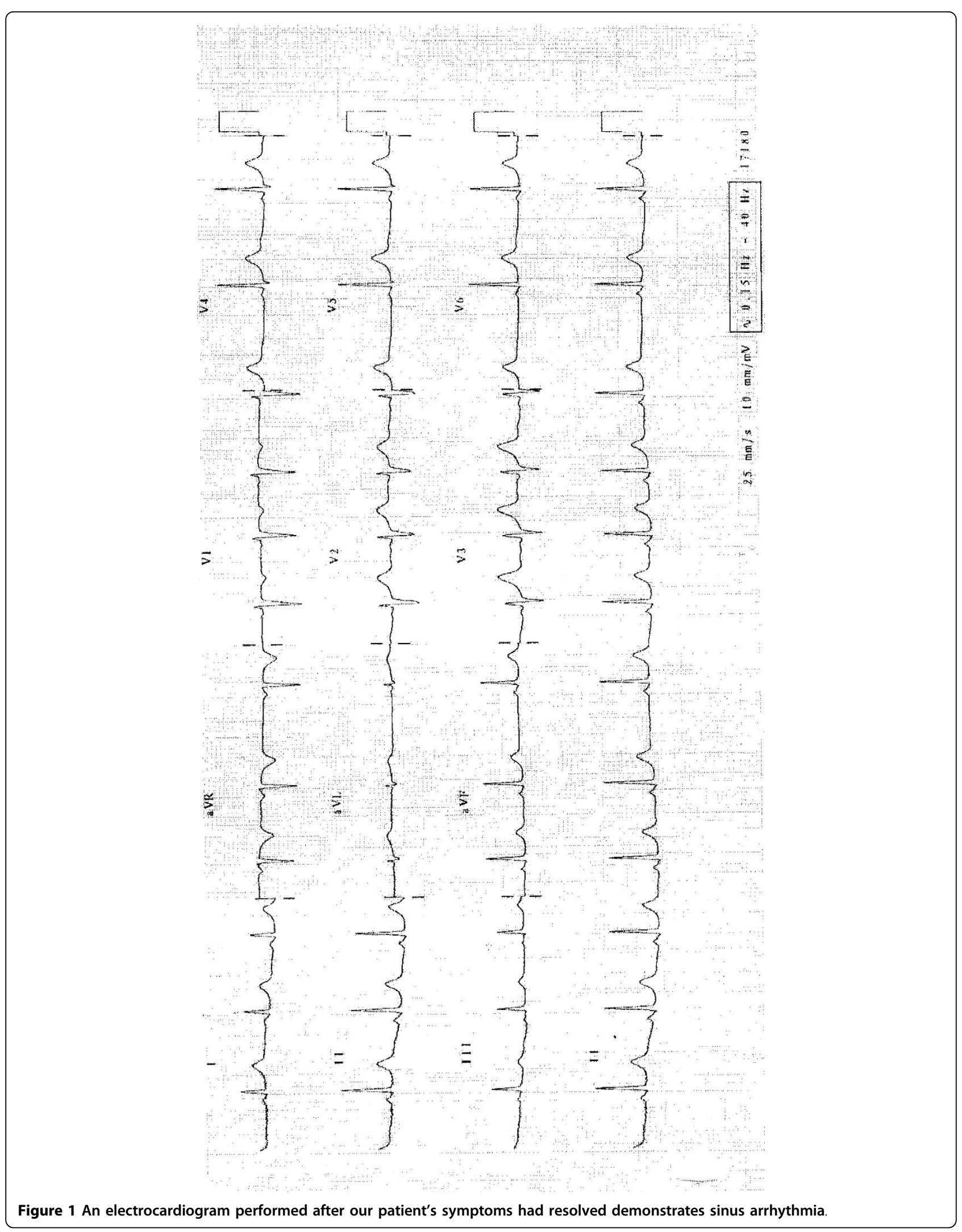




\section{Discussion}

Ibuprofen was the first non-aspirin NSAID to be approved for over-the-counter (OTC) use, and is widely considered to be the best tolerated drug of its class [1]. Previous studies [2,3] have demonstrated that adverse drug reactions (ADR) to NSAIDs predominantly involve the skin, the gastrointestinal tract, and the respiratory system. Although it had been previously suggested that ibuprofen may be involved in the development of ventricular arrhythmia [4], only recently has ibuprofen been demonstrated to produce arrhythmia both in vitro and in vivo (in guinea pigs) [5]. This is achieved by a dosedependent shortening of the cardiac action potential (AP), and shortening of the effective refractory period (ERP). This had resulted in a decrease of excitation propagation within the heart, which may provide substrate for an arrhythmogenic reentry circuit.

Cardiac arrhythmia secondary to ibuprofen overdose has been described in the medical literature. McCune and O'Brien reported the induction of atrial fibrillation (AF) in a previously-well 35-year-old man [6]. Meanwhile, Menzies et al. reported a case of broad complex tachycardia secondary to fulminant hyperkalaemia induced by ibuprofen overdose [7]. Interestingly, Gurfinkel et al. [8] have found that the intravenous administration of ibuprofen could immediately suppress arrhythmia during a cardiac electrical storm, but this work was conducted in the presence of decompensated cardiopathy, a condition not shared by the our patient. That our patient experienced only symptoms common to those associated with the sensation of palpitations, with no symptoms suggestive of an allergic aetiology, suggests that this case was not one of Kounis syndrome [9], which has recently been reported after routine ibuprofen use [10].

\section{Conclusion}

Our patient has suffered no recurrence of her symptoms, and in the absence of ibuprofen rechallenge, it is probable that standard dose ibuprofen was the causative link with her symptoms of palpitations. No firm conclusion can be drawn as to the underlying pathophysiological mechanism of her symptoms. This case appears to be the first case report in the medical literature of an event adverse to cardiac arrhythmia following standard oral ibuprofen administration.

Cardiac arrhythmia is a potentially fatal disorder that may exhibit heart palpitations as its initial (or only) symptom. The recognition of drug-induced cardiac disturbance is necessary to prevent the progression of symptoms to a more serious adverse event, and to avoid reexposing patients to ibuprofen with resultant recurrent cardiac arrhythmia. Further research is required to identify the pathophysiological mechanism of ibuprofeninduced cardiac arrhythmia.

\section{Consent}

Written informed consent was obtained from the mother of our patient for publication of this case report and any accompanying images. A copy of the written consent is available for review by the Editor-in-Chief of this journal.

\section{Acknowledgements}

The author would like to thank Tonia Mezzini for her assistance with the preparation of the manuscript.

\section{Competing interests}

The author declares that they have no competing interests.

Received: 22 October 2009

Accepted: 2 March 2010 Published: 2 March 2010

\section{References}

1. Moore N: Ibuprofen: a journey from prescription to over-the-counter use. I R Soc Med 2007, 100(Suppl 48):2-6.

2. Leroy S, Mosca A, Landre-Peigne C, Cosson MA, Pons G: Ibuprofen in childhood: evidence-based review of efficacy and safety. Arch Pediatr 2007, 14(5):477-484.

3. Titchen T, Cranswick N, Beggs S: Adverse drug reactions to nonsteroidal anti-inflammatory drugs, COX-2 inhibitors and paracetamol in a paediatric hospital. Br J Clin Pharmacol 2005, 59(6):718-723.

4. Pratt CM, Hertz RP, Ellis BE, Crowell SP, Louv W, Moyé L: Risk of developing life-threatening ventricular arrhythmia associated with tefenadine in comparison with over-the-counter antihistamines, ibuprofen and clemastine. Am J Cardiol 1994, 73(5):346-352.

5. Yang ZF, Wang HW, Zheng YQ, et al: Possible arrhythmogenic mechanism produced by ibuprofen. Acta Pharmacol Sin 2008, 29(4):421-429.

6. McCune $\mathrm{KH}, \mathrm{O}$ 'Brien $\mathrm{CJ}$ : Atrial fibrillation induced by ibuprofen overdose. Postgrad Med J 1993, 69(810):325-326.

7. Menzies DG, Conn AG, Williamson IJ, Prescott LF: Fulminant hyperkalaemia and multiple complications following ibuprofen overdose. Med Toxicol Adverse Drug Exp 1989, 4(6):468-471.

8. Gurfinkel EP, Guerlloy FP, Mautner B: Suppression of life-threatening tachyarrhythmias, and atrio-ventricular ischemic block following the administration of anti-inflammatory intravenous drug. Int J Cardiol 2007, 114(2):E56-E57.

9. Kounis NG, Zavras GM: Histamine-induced coronary artery spasm: the concept of allergic angina. Br J Clin Pract 1991, 45(2):121-128.

10. Kumar A, Berko NS, Gothwal R, Tamarin F, Jesmajian SS: Kounis syndrome secondary to ibuprofen use. Int J Cardiol. 2009, 137(3):e79-e80.

doi:10.1186/1752-1947-4-76

Cite this article as: Douglas: Palpitations following regular ibuprofen dosing in a 13-year-old girl: a case report. Journal of Medical Case Reports 2010 4:76. 